\title{
Aerodynamics and right-left symmetry in wind dispersal of maple, dipterocarps, conifers and some genera of apocyanaceae and magnoliaceae
}

\author{
Kirthi Tennakone \\ 13 Sheffield Drive, Peabody, Massachusetts 01960, USA.
}

\begin{abstract}
The wind dispersing seeds have evolved remarkable aerodynamic optimisation to minimise the speed of descent on detachment from the tree, so that they could be carried away by the wind and deposited a distance away from the parent. An efficient physical mechanism that enables slowing down the descent is rotation, which reduces the translational kinetic energy. Rotation and translation always define a right or left-handedness. The present work examines the aerodynamics of the dispersal of seeds of many species in relation to right-left asymmetry, giving examples to show that in nature this symmetry is broken either spontaneously or intrinsically. In the former situation, seeds have no geometrical right-left asymmetry, but an initial instability chooses one sense of rotation (right or left). Whereas in the latter, geometrical asymmetry dictates the sense of rotation. Seeds of the maple familiy belongs to the first category and a theortical model is presented to explain the motion. Seeds of dipterocarps, conifers and some genera of apocyanaceae and magnoliaceae are found to possess a handedness, which determines the rotation during seed fall. In dipterocarps, conifers and magnoliaceae, both right-handed and left-handed seeds are produced in the same tree but correlated to the handedness of the sprial phyllotaxy of the branch that bears the fruit. Apocyanaceae is found to be unique and seeds of all genera have the same handedness. The details of extensive field observations, experiments and theoretical interpretations are presented to illustrate the relationship of right-left asymmetry to the aerodynamics of seed wind dispersal, pointing out the implications of evolutinary optimisation in practical aerodynamics.
\end{abstract}

Keywords: Apocyanaceae, dipterocarps, magnoliaceae, maple, plant handedness, seed aerodynamics.

\section{INTRODUCTION}

In certain plants wind is an effective mode of seed dispersal. Prior to evolution of birds, wind dispersal could have been the sole mode of long distance seed distribution on land. Consequently, trees adopting wind dispersal have evolved seeds with intricate aerodynamic appendages to slow down the descent, enabling them to be transported by wind (Greene \& Quesada, 2005; Pandolfi \& Izzo, 2013; Stevenson et al., 2015). Plumes attached to lighter seeds achieve this via increase of the air drag. However, heavier seeds storing food for the initial phase of germination cannot utilise this method and the viable physical alternative would be to make them spin while falling. Here the gravitational potential energy of a falling seed dissipates largely via transformation into rotational kinetic energy, effectively inducing an aerodynamic lift.

Two of the most diverse and widely distributed angiosperm tree genera are maple (Aceraceae) and dipterocarps (Dipterocarpaceae). Former is one of the most common tree species in temperate zones while the latter dominates rain forests in the tropics (Ashton \& Gunatilleke, 1987; Corlett \& Primack, 2005; Gunatilleke et al., 2006; Lamarque, 2013). Their success is due to bearing of auto-gyrating seeds. Pines and other conifers (Coniferae) distributed worldwide were the dominant tree species in the Mesozoic era. Then their predominant mode of dispersal may have been the wind (Stevenson et al., 2015), and present day conifer seeds carry a trait for auto-gyration. 
Spinning and translational motion of an object involves a handedness. Something falling down and rotating clockwise when viewed from above differs from the same rotating counter-clockwise. A maple samara dropped from a height rotates either clockwise or counterclockwise. Although there are reports in literature discussing kinematics of maple seed motion (Lentink et al., 2009; Varshney et al., 2012; Sohn et al., 2014), to the knowledge of the author, the aspect of handedness of motion has not been investigated. Observations indicate that maple samaras or any of the macroscopic organs of maple trees have no handedness (right-left distinguishing property) attributing maple seed rotation to a spontaneous parity violation (right-left symmetry). In contrast, the dipterocarp fruit and other organs of their trees possess handedness and the sense of rotation of the fruit during falling is predetermined. Dipterocarp trees have distinct right-handed (RH) and left-handed (LH) twigs, flowers and fruits. Strangely the extensive literature on taxonomy of dipterocarpaceae (Foxworthy, 1911; Ashton, 1980; Smitinand et al., 1980; Simmathri \& Turnbull, 1998) does not record observing of this property. Pines and magnolias are also found to have handedness attributes similar to dipterocarps, whereas the entire family of apocyanaceae is ambidextrous.

The investigation reported here based on extensive observations on symmetries in plants theoretically analyses the aerodynamics of wind dispersal in relation to right-left symmetry. Detailed observation of the morphogenetic features of seeds as well as parent trees and simple experimentation illustrate how evolution has optimised the wind dispersal strategy.

\section{METHODOLOGY}

The analysis and discussion is based on: (a) field observations and sample collection, and experimentation with wind dispersing seeds; (b) examinations of the organs of plants (flowers, leaf and branching arrangement) that bear these seeds to ascertain whether right-left asymmetries exist; and (c) experimentation with paper toy models, so that parameters can be readily adjusted.

(a) Wind dispersed maple samaras were collected from Peabody area, Massachusetts, United States. Seeds of Sugar maple (Acer saccharum Pax), Norway maple (A. platanoides L.), Sycamore (A. pseudoplatanus L.), Red maple (A. rubrum), Silver maple (A. saccharinum Pax) and Box Elder (A. negundo L.) often mixed up were separated and stored at ambient conditions $\left(\sim 15{ }^{\circ} \mathrm{C}\right.$, relative humidity $\left.\sim 60 \%\right)$. The samaras of the first 4 varieties have close similarities compared to the $5^{\text {th }}$ and $6^{\text {th }}$ that are lighter and differ in wing and seed geometry. Therefore, most experiments were conducted with Norway maple and Box Elder samaras. Average weights were determined from 300 samples, and 100 samples were used for determination of the dimensions. Wing structure was examined by low-power microscopy before and after chemical treatment with acetone and hypochlorite. These treatments enhanced the transparency of wings to examine the veins. The position of the centre of gravity was assessed by balancing the samara on a vertically placed horizontal edge both length-wise and breadth-wise. Physical alterations to the samaras were made by cutting the wings and seed edges with scissors, sanding the seed surfaces symmetrically or asymmetrically, and coating with paraffin wax and polishing. Terminal velocities were determined by releasing the seeds from varying heights and timing the motion for the final $2-3$ metres. To fix the sense of rotation (relation to the downward vertical), a region in the seed surface was lightly painted. As leading edge is the ridge over the wing, observing the uppermost side enables fixing the sense of rotation. Each seed was dropped 100 times from a height of $\sim 3 \mathrm{~m}$ to assess the probability of the either sense of rotation. The effect of the positioning of the seed on releasing was carefully observed. Before and after each physical modification the average: (1) distance of fall at which spinning starts, (2) terminal velocity, and (3) sense of rotation were assessed. The effect of drying the seed as a whole or exposing one surface to the sun was also examined. Twins in one fruit were examined to determine whether there exist any correlations of sense of rotation.

Dipterocarpus zeylanicus samples were collected from Gampaha, Kurunegala, Kalutra, Matara and Kegalle Districts of Sri Lanka. Terminal velocities were ascertained by dropping the fruits from $20-30 \mathrm{~m}$ height. The angular speeds of falling fruits through height of $\sim 10 \mathrm{~m}$ were estimated by stroboscopic measurements. Wing surface was examined by magnification and the angles between the longitudinal veins were measured after imprinting the vein structure on paper by pressing the inked wings. The nut was cut into transverse sections to determine the geometry of sepal arrangement. Wing and seed modification was done in the same way as for maple.

(b) Whenever an opportunity arose, observations were conducted during the past 40 years to ascertain the handedness of the seeds and sense of spinning to determine whether seed asymmetries transcend to other organs. The asymmetries in flowers, fruits, leaves and 
branching arrangement were qualitatively examined. The relationship of fruit handedness to that of flower and leaf phyllotaxy in dipterocarps and related species was extensively investigated. Almost all the genera of apocyanaceae in Sri Lanka (wild and ornamental) were examined to identify traits of handedness in phyllotaxy, flowers, fruits and seeds. Two Sapu (Michelia nilagrica) varieties growing in the Central Province of Sri Lanka, and ornamental magnolias were used to ascertain the relationship of leaf phyllotaxy to flower and fruit morphology.

Observations and experiments were also conducted to ascertain whether the handedness of the fruit or sense of rotation was affected by the individual tree or its branch and flower, geographic location or genetic variations and whether the handedness of the nut passes to the seedling. Seeds of hibiscus varieties and papaya from right or lefthanded plants were germinated and handedness of the progeny was recorded.

(c) Toy models of systems that rotate during falling were constructed out of paper to simulate seed wind dispersal mechanisms and illustrate how asymmetry imparts rotational motion, slowing down the rate of fall.

\section{RESULTS AND DISCUSSION}

\section{Maple samara characteristics}

The maple fruit is a bilaterally symmetric structure with two conjoined winged seeds (samaras), which are distinctly separated from each other and the stalk as the fruit ripens and dries (Figure 1). Generally, wind dispersal begins in late summer and continues into autumn, and the pattern varies from species to species. The median weight

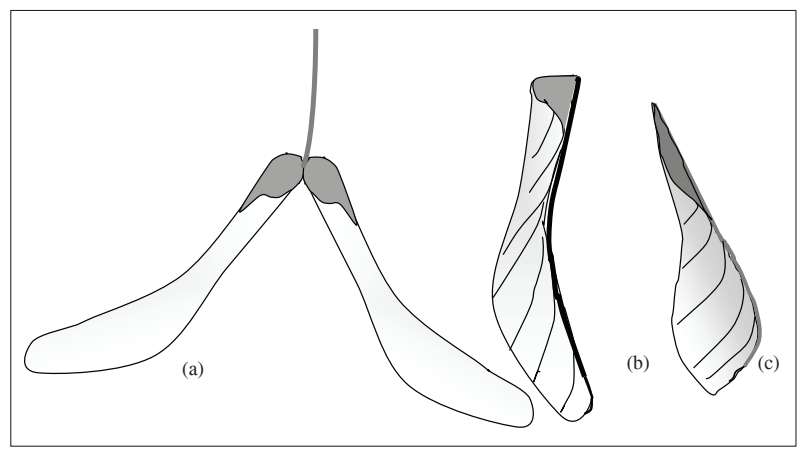

Figure 1: (a) Schematic diagrams of (a) a fruit of Norway maple showing two conjoined samaras, seeds and the stalk; (b) wing shape and veins in Norway maple; (c) wing shape and veins in Box Elder and dimensions of a maple samara varies from species to species and perhaps climate conditions. Typically, a Norway maple samara has a length $\sim 4.5 \mathrm{~cm}$, maximum breadth $\sim 1.5 \mathrm{~cm}$ with a $\sim 6 \mathrm{~mm}$ seed and a median weight $\sim 140 \mathrm{mg}$, of which more than $\sim 75 \%$ is contributed by the seed and the thick ridge of vein supporting the wing (Figure 1). The Box Elder samaras have smaller dimensions; length $\sim 3.8 \mathrm{~cm}$, maximum breadth $\sim 1 \mathrm{~cm}$. They are also lighter with a median weight $\sim 75 \mathrm{mg}$ with an elongated seed carrying about $80 \%$ of the weight. The wings of both varieties taper in breadth-wise direction towards the lobe supported by the hard and relatively thick ridge (Figure 1). A distinctive characteristic of the two varieties is the geometry of the seed and the ridge. The Norway maple seed has an oval shape with an apex pointing towards the ridge, whereas the Box Elder seed is elongated with pointed apexes on both sides (Figure 1). If the two types are held, with wings pointing vertically downwards, the ridge appears concave in the case of Norway maple and convex in Box Elder (Figure 1). The centre of gravity of Norway maple and Box Elder samaras resides $\sim 2-4 \mathrm{~mm}$ from the seed apex. Both sides of the wing surfaces are covered with a pattern of striations as shown in Figures 1 (b) and (c), but more densely populated than in the depiction. The texture and pattern of distribution on either side being identical as they originate from veins embedded in the middle of the wing tissue. The surface also possesses the property of not retaining water. Otherwise the expected function could not be performed effectively.

\section{A theoretical formulation of maple seed motion}

A maple samara falling under the gravity starts spinning, slowing down the descent, enabling it to be carried away by wind to further locations. They rotate about a point located near the seed very close to the centre of gravity, while the plane of the wing stays slightly inclined to the horizontal. The motion and mechanism of slowing down during descent involve intricately complex aerodynamics owing to nonlinearity of autorotation (Lugs, 1983). A simple explanation follows from consideration of energy conservation. When a samara of mass $m$ falls down a vertical distance $h$ and acquire translational and angular velocities $v$ and $\omega$ respectively, the change in potential energy $\mathrm{D} V=m g h$, needs to be always greater than the kinetic energy acquired DT $=1 / 2\left(m v^{2}+I \mathrm{w}^{2}\right)$, where $I=$ moment of inertia of about a vertical axis through the centre of mass. This is because, a part of potential energy transforms into kinetic energy of air set into motion, and a part dissipated as heat generated due to the air resistance. Thus we obtain the inequality,

$$
v<\sqrt{\left[2 g h-(I / m) \omega^{2}\right]}
$$


In equation (1), $\sqrt{ }(2 g h)$ is the velocity acquired in the absence of spinning action and air resistance. Rapid rotation, large moment of inertia and smaller mass all favour lowering of the time of descent. Detailed analysis of the problem requires an understanding of the aerodynamic lift and torque forces arising from fluid motion and drag. These forces depend on the dimensions, shape, and nature of the surface, mass and its distribution and elastic properties.

The structural architecture makes a samara mirror symmetric in the sense if it is laid down flat on a table and turned upside down; the shape appears exactly the same as the mirror image. Similarly in the maple fruit, the twins are mirror images of each other. This symmetry implies that they do not possess handedness. Imagine a samara released from the rest keeping the wing in a vertical plane. One could argue that in this situation it cannot auto-rotate during falling, because in the absence of a right-left distinguishing feature, there is no way to decide which way to turn and rotate. However, an alternative way of preserving right-left (R-H) symmetry exists. If the object rotates clockwise and counter-clockwise with equal probabilities, the required symmetry would be again preserved. Frequently, the symmetries in nature are respected in this manner; although individual symmetry violations exist, they occur in equal probabilities. This is exactly what we observe in dropping a maple seed.
For a while it moves without rotating in an orientation where the heavy seed points downwards. Subsequently, the movement turns erratic followed by an orderly rotation in either clockwise or counter-clockwise sense (Figure 2). Transition occurs when the velocity of the centre of the mass reaches a critical value $\left(v_{\mathrm{C}}\right)$. The transition process needs to conserve energy, and angular and linear momenta. If a distributed and massive body takes up linear and angular momenta (in this case stream and vortices of air), the kinetic energy transferred to it would be relatively small. If the transition is assumed instantaneous and resistive loss is minimal, conservation energy yields,

$m g h \leq 1 / 2 m v_{C}^{2} \leq 1 / 2 I \omega_{C}^{2}$

where $I=$ moment of inertia of the about the centre of gravity (object rotates about vertical axis inclined at a small angle to the horizontal axis). On releasing a samara from the rest, a sudden decrease in the translational velocity below $v_{\mathrm{C}}$ is noticeable at the point rotation ensues. Since angular momentum needs to be conserved, irrotational air flow invariably turns rotational. The system has all the characteristics of a spontaneous breaking of symmetry (SBS) sometimes seen in fluid motion (Zhang \& Childress, 2005; Bagheri et al., 2012). Here an unstable initially symmetric state suddenly develops into one of the asymmetric states, when a

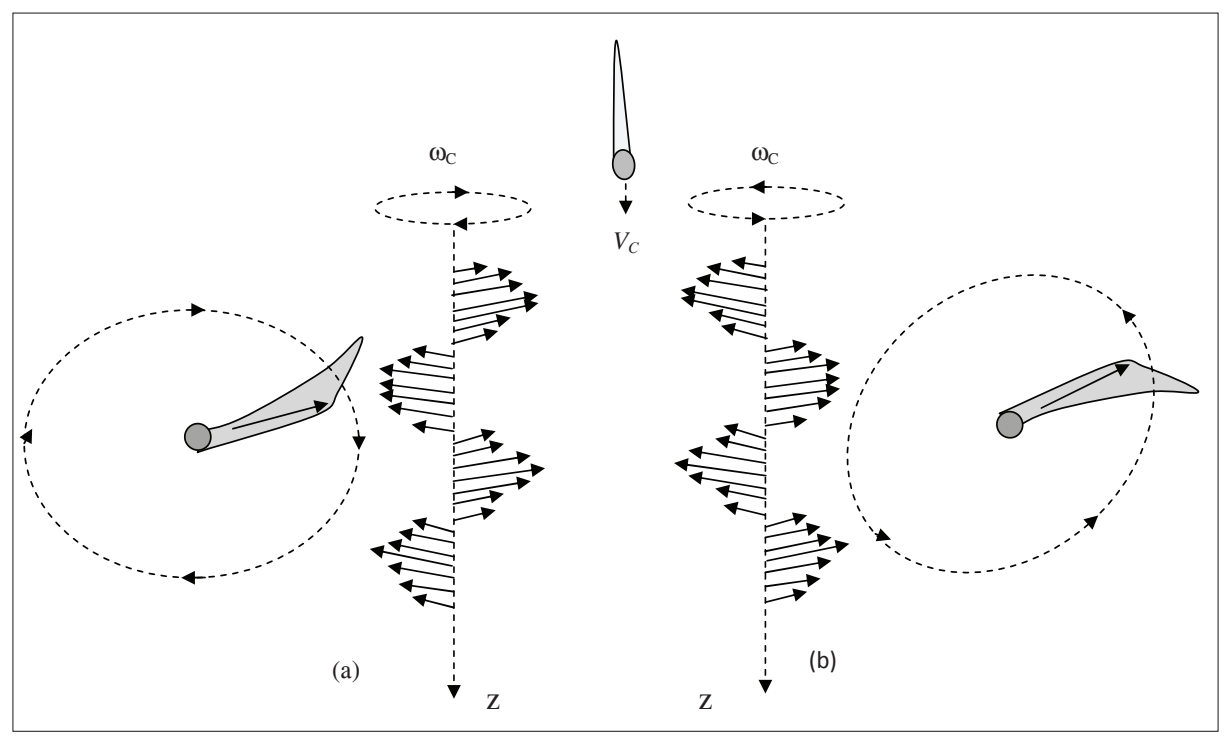

Figure 2: Schematic sketch illustrating (a) clockwise; (b) counter-clockwise spiraling of a falling maple samara once it reaches the critical velocity (VC). The pattern of lines drawn perpendicular to vertical axis (z-axis) are the projections of the arrow marked in the samara on a plane passing through the $z$-axis and circles indicate the appearance and the sense when viewed from above. The darker shading of the samara corresponds to its thicker region. During motion thicker edge is always the leading edge. 
crucial parameter reaches a critical value; the inherent symmetry being conserved in realising different allowed states with equal probabilities.

Figure 2(a) and (b) give a schematic representation of the clockwise and counter-clockwise downward spiraling of a maple samara. Arrows in these figures point away from the seed towards the opposite end of the wing representing the position of the wing. In both clockwise and counter-clockwise motions, the thicker edge is always the leading edge. Shading indicates the thickness variation across the wing, the darker edge being the thickest $(0.3 \mathrm{~mm})$ and the wing tapers down $(\sim 0.01$ in a wing breadth $\sim 1 \mathrm{~cm}$ ) just like an aerofoil. As transverse cross-sections of the wing are nearly symmetrical, little lift is generated at zero angle of attack.

The involvement of translation as well as rotation and associated lift and drag forces complicates the motion of falling maple seeds defying rigorous analysis based on first principals. Even the uniform rotation of a rigid propeller pointing to a fixed direction offers intricate complexity, and solutions sufficiently precise for application has been arrived, incorporating empirical inputs. The following simplified treatment presented in the next paragraph explains the salient features of this intriguing system.

Forces acting on an air foil can be related to force acting at a point and a couple, which changes with the angle of attack. However, there exists one particular point, the so-called aerodynamic centre $\mathrm{C}$, around which the magnitude of the couple remains independent of the angle of attack (Carlton, 2007; Pope, 2010). Forces acting on a falling maple samara are: the aerodynamic force $A$ (the resultant of drag and lift forces) with components $A_{\mathrm{Z}}=$ lift force, $A_{\varphi}=$ the azimuthal torque

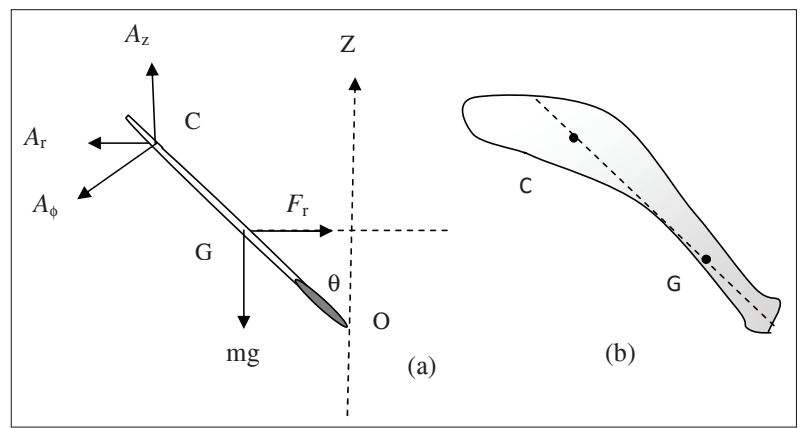

Figure 3: (a) Schematic representation of the forces acting on the samara: centre of gravity, aerodynamic centre and centre of rotation are marked as $\mathrm{G}, \mathrm{C}$ and $\mathrm{O}$, respectively; (b) positioning of the points $\mathrm{G}$ and $\mathrm{C}$ on the surface generating force and $A_{\mathrm{r}}=$ radial component, all acting at $\mathrm{C}$, and weight $=m g$. For equilibrium of rotation, a centrifugal force $F_{\mathrm{r}}=m \omega^{2} b \operatorname{Sin} \theta$, acting at the centre of gravity $\mathrm{G}(\mathrm{OC}=a, \mathrm{OG}=b$ and $l=$ length span $)$ needs to be provided aerodynamically. The centre of rotation $\mathrm{O}$ is not exactly about centre of gravity. The torque necessary for rotation around the z-axis, against provided by $A_{\varphi}$. The wing is held in an inclined position (an angle $\theta$ to the vertical axis) by these forces.

The lift forces operate normal fluid flow and drag forces opposite to the direction of the flow. Both these forces are proportional to the square of the radius of the blade and square of velocity, so that,

$A_{z}=C_{z L} l^{4} \omega^{2}-C_{z D} l^{2} v^{2}$

$A_{\varphi}=C_{\varphi L} l^{2} v^{2}-C_{\varphi D} l^{4} \omega^{2}$

$A_{r}=C_{r L} l^{4} \omega^{2}$

where $C_{L}$ and $C_{D}$ with subscripts $\mathrm{L}$ and $\mathrm{D}$ denote the respective lift and drag coefficients (proportionality of these forces to fluid density has not been incorporated explicitly thus they have dimensions of a density). As there is no translational motion in $r$ direction, balancing of forces in the radial direction require,

$A_{\mathrm{r}}=F_{\mathrm{r}}=m \omega^{2} b \operatorname{Sin} \theta$

The force $F_{\mathrm{r}}$ is generated aerodynamically and compensates centrifugal acceleration. From equations (5) and (6) it follows:

$\operatorname{Sin} \theta=\frac{C_{r L} l^{4}}{m b}$

Thus writing $A_{\mathrm{r}}$ in the form in equation (5) is in agreement with the observation that inclination of the wing to the vertical direction remains constant throughout motion.

Taking moments of forces about $\mathrm{O}$, the instantaneous centre of rotation, we obtain the following equation of motion,

$I_{O} \frac{d^{2} \theta}{d t^{2}}=A_{r} b \operatorname{Cos} \theta-A_{z} a \operatorname{Sin} \theta+m g b \operatorname{Sin} \theta$

Thus the equilibrium occurs at $\theta=q_{0}$

$\operatorname{Tan} \theta_{0}=A_{r} / A_{z}=C_{r L} l^{4} b \omega^{2}\left[\left(C_{z L} l^{4} \omega^{2}-C_{z D} l^{2} v^{2}\right) a-m g b\right]^{-1}$ 
As the drag forces in the propeller are largely azimuthal, opposing rotation rather than translation, the vertical motion experience little drag force and dissipation results mainly from rotation. In this situation $C_{z L} l^{2} \omega^{2}>C_{z L \mathrm{D}} v^{2}$ and equation (9) can be approximated as,

$\operatorname{Tan} \theta_{0} \approx C_{r L} l^{4} b \omega^{2}\left[\left(C_{z L} a l^{4} \omega^{2}-m g b\right]^{-1}\right.$

Thus a necessary condition for equilibrium of the object in the orientation shown in Figure 3(a) is,

$\omega^{2}>\frac{m g b}{C_{z L} l^{4} a} \quad(b \neq 0)$

If the cone angle $\theta$ is perturbed by a small amount $\varepsilon$, inserting $\theta=q_{0}+\varepsilon$ in equation (8), we obtain an equation of the form $d^{2} \varepsilon / d t^{2}=-k \varepsilon(k>0)$ indicating that equilibrium resist perturbations. Equation (11) explains that auto-gyration could occur only if the angular velocity exceeds a critical value. This is experimentally observed; samara starts spinning only after falling through some distance $h$. From equation (2), the maximum angular velocity attainable is $(2 m g h / I)^{1 / 2}$, giving $h>b I\left[2 C_{\mathrm{zL}} a^{4} l\right]^{-1}$.

Motion in the vertical direction is described by,

$m \frac{d^{2} z}{d t^{2}}=A_{z}-m g$

Thus the equation of motion for rotation can be expressed as,

$I_{\phi} \frac{d \omega}{d t}=(a+b) \operatorname{Sin} \theta_{0}\left(l^{2} C_{\phi L} v^{2}-C_{\phi D} l^{4} \omega^{2}\right)$

where $I_{\varphi}=I_{\mathrm{O}} \operatorname{Cos}^{2} \theta=$ moment of inertia of inclined seed about the axis of rotation. The falling seed soon approaches the terminal translation and angular velocities $v=v_{\mathrm{T}}, \omega=\omega_{T}$, which are the parameters relevant to wind dispersal. The terminal values correspond to setting the time derivatives in equations (12) - (13) equal to zero, i.e.,

giving,

$A_{z}-m g=0$

$C_{\phi L} v_{T}^{2}-C_{\phi D} l^{2} \omega_{T}^{2}=0$

$\omega_{T}^{2}=\frac{m g}{C_{z L} l^{4}}$
$v_{T}^{2}=\left[\frac{C_{\phi D}}{C_{z L} C_{\varphi L}}\right] \frac{m g}{l^{2}}$

From equations (7), (10) and (11) we obtain,

$\operatorname{Cos} \theta_{0}=\frac{g a}{\omega_{T}^{2} b l}$

The parameters $\theta_{0}, \omega_{\mathrm{T}}$, and $v_{\mathrm{T}}$ are readily measurable, the typical values for Norway maple being $\theta_{0} \sim 68^{\circ}, \omega_{\mathrm{T}} \sim$ $13 \mathrm{~Hz}, v_{\mathrm{T}} \sim 1 \mathrm{~ms}^{-1}$. Experiments show that the cone angle $\theta_{0}$ is not highly sensitive to $m$ as seen from equation (18). From the same equation, we obtain $l(b / a) \sim 1.6 \mathrm{~cm}$, giving $b / a \sim 0.4$. This factor defines the position of the aerodynamic centre relative to the centre of gravity. Again from equation (17) for a typical value of $l \sim 3.8 \mathrm{~cm}$, we obtain the lift to drag ratio $C_{\varphi \mathrm{L}} / C_{\varphi \mathrm{D}}=9.4$. This ratio is significantly high, e.g., compared to that of a helicopter. The average terminal velocity $\left(\sim 0.6 \mathrm{~ms}^{-1}\right)$ is slower in Box Elder samaras compared to Norway maple. A more striking difference is the time taken to attain the terminal velocity, which is less than half the time taken by Norway maple. The elongated seed of Box Elder reduces its moment of inertia. Box Elder is a shorter tree $(\sim 20 \mathrm{~m})$ compared to Norway maple $(\sim 50 \mathrm{~m})$. It is hard even to speculate the secret of the difference of their wing shapes (Figure 1).

Wind tunnel experiments have shown that in a spinning maple samara, vortices are developed at the leading edge, greatly increasing the lift (Hederstrom, 2002; Sane, 2003; Lentink et al., 2009). Possibly the wing striations play a crucial role in confining the vortex to the leading edge and facilitate streamlines to leave the trailing edge smoothly. Striations are smoothed out by coating paraffin wax and polishing, and $v_{\mathrm{T}}$ increases drastically. If the treatment is given to one wing surface, the seed gyrate with the untreated surface upwards, suggesting that the striations have an influence in enhancing lift forces. Possibly they act as vortex confiners as well as generators. The purpose of the veins is not only to fix the structure of the wing but also to serve an even more important aerodynamic requirement.

Simple experiments conducted with Norway maple and Box Elder seeds support the validity of the relationship in equation (17), if $l$ is taken as the maximum length of the wing. On reducing the wing area by cutting the wing breadth-wise, terminal velocity rapidly decreases. If the reduction is length-wise, up to a point, the terminal velocity decreases more slowly. It 
appears that the sole criterion of wing design is not only minimisation of terminal velocity but also propelling along the wind direction. If maple seeds are dropped from some height at the time of a blowing wind, they sometimes move up or down in wide helixes as eddies energise motion. Possibly they are wondering in von Karman streets (Narasimhan, 2015) as wind blow past an obstacle.

A question also arises regarding the stability of the wing against rotations about the length-wise axis GC of the wing. In a symmetrical wing (transverse cross-section of Maple samara is symmetrical), the point of action of the position of the aerodynamic centre remains unaltered irrespective of changes in the attack angle. Therefore the moment forces about the axis GC is zero, implying that the move about axis is in neutral equilibrium. Thus, the system could adjust to maintain the angle of attack optimally.

The importance of right-left asymmetry in wind dispersal of seeds

Wind dispersal necessitates slowing down of the rate of fall of the seed, so that wind could carry the seed further away from the parent tree. The physical principle that enables optimising this strategy is generation of rotation so that transitional velocity of the centre of mass is continuously reduced. The additional advantage of rotation is aerodynamic lift, opposing gravity which further reduces the rate of fall. Rotation and the direction of transitional motion define a right or left handedness. Such a handedness can be achieved either spontaneously or intrinsically. In the former situation, the object (seed) possesses no geometrical right-left asymmetry, but one sense of rotation is chosen as a consequence of the aerodynamics resulting from the design of the seed. In the latter case the seed itself possesses a geometrical right-left asymmetry giving preference to one sense of motion. It is amazing to observe that both these principles are adopted in nature. The discussion below presents examples of two cases.

\section{Right-left symmetry of falling maple seeds}

Most symmetries in living and non-living systems including elementary constituents of matter, are not perfectly realised giving rise to frequent instances of biasing. The same feature is seen in the sense of rotation of maple samaras. As discussed earlier maple seeds possess no chirality to decide a sense of rotation when falling, nevertheless they auto-rotate as a result of spontaneous breaking of the R-H symmetry. The required symmetry would be respected if both senses of rotation happen with equal probabilities. In dropping a samara from a fixed height, a large number of times, a higher probability of one sense of rotation is frequently observed. If sizeable samples are examined this way, the histogram obtained corresponds to a normal distribution, concluding that there exists no intrinsic biasing towards clockwise or counterclockwise, and the observed individual violations happen as effects of accidental influences operating without a universal bias. As only R-L distinguishing characters can introduce a bias, we performed a number of experiments to identify the factors that could introduce such biases.

(i) In maple samaras the embedded seed symmetrically protrudes above the wing surface on both sides. We gradually sanded the protrusion on one side and observed the sense of spinning. When this is done, two right-left distinguishable objects are obtained (Figure 4). Now the object (b) cannot be superposed on (a) by translations and rotations. If they are dropped from a height, during descent the sanded surface tends to remain uppermost. As leading edge is the one with the ridge, (a) prefers to rotate clockwise and (b) counter-clockwise because of the centre of gravity bias. The bias increases with the degree of sanding. When the seed protrusion is fully levelled, the probability for this trend is about $60 \%$ for Norway maple and $75 \%$ for sugar maple.

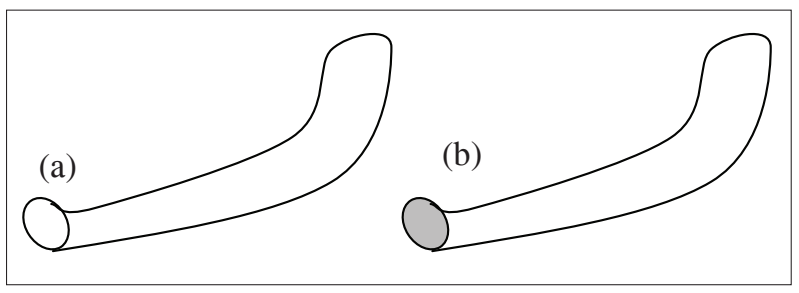

Figure 4: Two maple samaras where the protruding seed on one side has been sanded off (a) sanded seed on the upper surface; (b) sanded seed backside

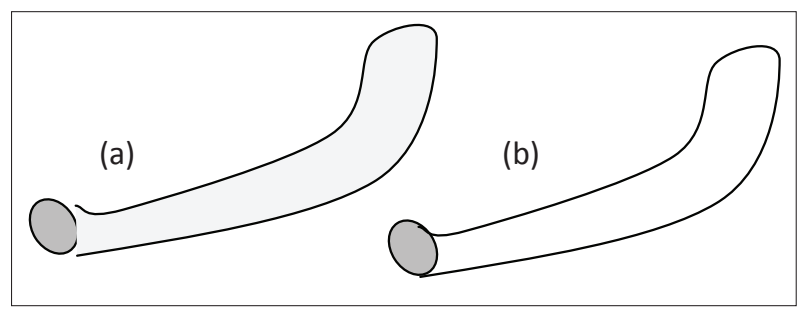

Figure 5: Two maple samaras where one wing surface has been polished by rubbing paraffin wax (a) upper surface polished; (b) backside polished 
(ii) In another experiment one wing surface was lightly coated with paraffin wax and polished to level striations. Figure 5 indicates two samaras laid on a table; (a) upper surface is polished and (b) the backside is polished. When they are dropped, (a) preferentially spins counterclockwise and (b) clockwise. As the leading edge is the ridge, the result implies that they prefer to spin, facing the un-polished surface upwards. We confirmed that the effect was not caused by the increase of weight. Even if a portion of the seed surface on the opposite was scraped to compensate for any weight gain, the trend continued.

The observation suggests that interfering with striations affect the lift, and they seem to play an important role in vortex formation and attachment.

(iii) A bias in initial conditions will give a preference to the sense of spinning. If the samara is held with the plane of wing horizontal, and given a torque to turn the leading edge forward, the observed trend will be to continue spin in the sense induced.

A system governed by dynamics of SBS displays very high sensitivity even to minutest biases near the point of criticality. The observed biases in individual seeds originate from accidental influences. The maple tree has no signs of R-L asymmetries. At this scale of phenomena, the Corolis force would not have an effect.

(iv) The senses of rotation of the twins of the twin samaras show a slight opposite correlation. This can be understood as drying and action of light could be different on the faces of the fruit.

\section{Right-left symmetries in plants and the fascinating Dipterocarpus}

Not only the fruit and the samaras, maple trees have no macroscopic organs carrying an attribute of handedness. A majority of plant species falls into this group. Here the leaves and their phyllotaxy, the pattern of branching or flowers and fruits display no R-L distinguishable characters. After extensive observations of symmetries in plants, the author noticed that plants more primitive in the phylogenetic ladder and the ones that mature fast possess R-L symmetries to a higher degree. There seems to be a universal trend in all systems that, as complexity increase and when they adapt to perform more functions, the symmetries disappear. Some plants demonstrate a unique handedness in one or more of their organs as a genetically inherited quality common to the species. The author noticed that in apocyanaceae, flowers in all genera and species imbricate in the same sense- making the whole family left-handed. This may be an irrevocable genetic accident of no value to the plant. There are also species having individuals of either handedness, classifiable as RH and LH members. Having examined hundreds of plants falling into this category we found that if they propagate from seeds, the RH and LH individuals occur in equal $(50 \%)$ probabilities. Here the cause seems to be a result of the dynamics of morphogenesis. During cell proliferation, instability sets in and the plant has to spiral either $\mathrm{R}$ or $\mathrm{L}$ with respect to the direction of growth- a case of spontaneous breaking of $\mathrm{R}-\mathrm{H}$ symmetry. We have observed that the phyllotaxy of leaf arrangement in un-branched papaya (Carica papaya L.) is either R- or L- handed. Lateral branches, if they grow, favour the handedness of the stem but reversals also occur. When papaya seeds germinate the first few leaves show no spirality, but as the growth progresses, they follow a pattern of either R or L helicity. There are also species and entire families where many of the organs possess a handedness. However in a matured plant of this class, RH and LH organs bear in equal probabilities. The plants of malvaceae generally have branches or extended regions in a branch where the leaf arrangement shows a handedness, and the sense of imbrication of petals in flowers correlating with the handedness of the parent branch (Tennakone et al., 1982). The feature displays more conspicuously in herbs belonging to the family or young plants. Here the plant itself possesses a handedness, because it bears more branches and flowers of one kind ( $\mathrm{R}$ or $\mathrm{L}$ ) than the other. As the plant matures, it gradually sheds the handedness in bearing organs of both types in equal numbers. The R-L distinction to us humans is of paramount importance. We would not be able to engage even in routine matters without this ambidexterity. Although macroscopic R-L disparities originate in plants as genetic accidents or consequences of morphogenesis, it is hard to see any advantages for survival. The exception seems to be the use of this trait as an advantage for wind dispersal.

Plants of dipterocapaceae and families in the order malvales to which they allies (Dayanandan, 1999) endow the differentiated organs with these peculiar characteristics. The R-L symmetry seen in dipterocarps is rarely seen in other families. They have spiral phyllotaxy and shoots with $\mathrm{R}$ and $\mathrm{L}$ leaf spirals distributed in equal probabilities, and induce this symmetry to other organs developed in the twig. In the discussion, the convention used to define $\mathrm{R}$ and $\mathrm{L}$ will be as follows: if a shoot is held vertical with the apex upwards and one leaf apex pointing the observer, the spiraling is defined as R (L), if the next leaf just above places itself towards $R(L)$. The same will be adopted to assign the handedness to the arrangement of petals and sepals in a flower. In most dipterocarps, flowers bear as axial racemes 
popping out at the leaf brackets (Figure 6). As a result the axles of racemes contain the same spirally. Again spirality appears in the setting of flower buds on the raceme axle (Figure 6). The handedness of this spiral changes alternatively; if the pattern of flower buds in one raceme is right, the ones above and below are left. Progression of spirals continues to other organs. The sepals and petals contort and imbricate in the same sense, defining a handedness. Here again, the sense of the spiral alternates from one flower bud or flower to the next. In a majority of dipterocarps all sepals do not develop equally, and a few elongate as wings attached to the nut (Figure 6). Generally two sepals standing at angles $144^{\circ}$ of a pentagon elongate concomitantly as the fruit grows, greatly shunting the growth of the others. Nevertheless, the original handedness of the sepals retains, endowing a right or left handedness to the fruits (Figure 7). Thus wings shaped as RH or LH propellers fall rotating in opposite senses. A propeller without a handedness cannot rotate and propel. We observed that there is no detectable disparity in the abundance of $\mathrm{RH}$ and $\mathrm{LH}$ nuts in an individual tree of Dipterocarpus zeylanicus or

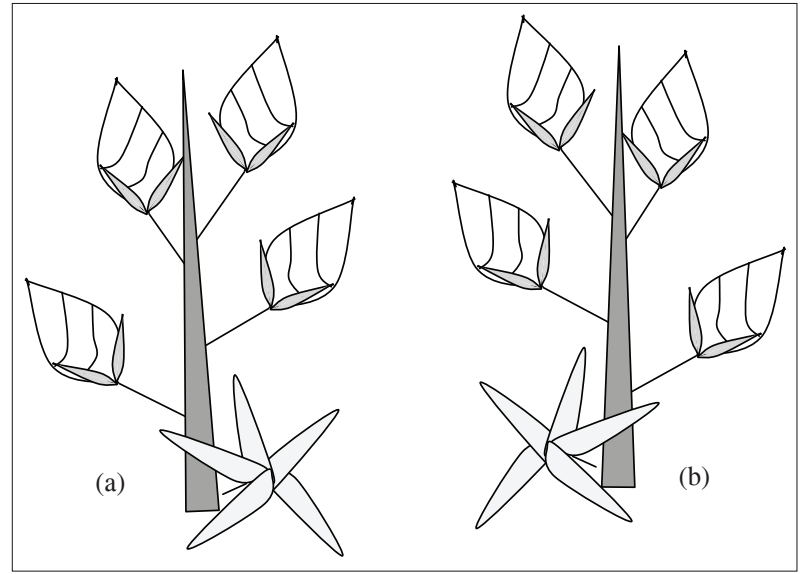

Figure 6: Right-handed (a) and left handed (b) axle racemes of a typical dipterocarp

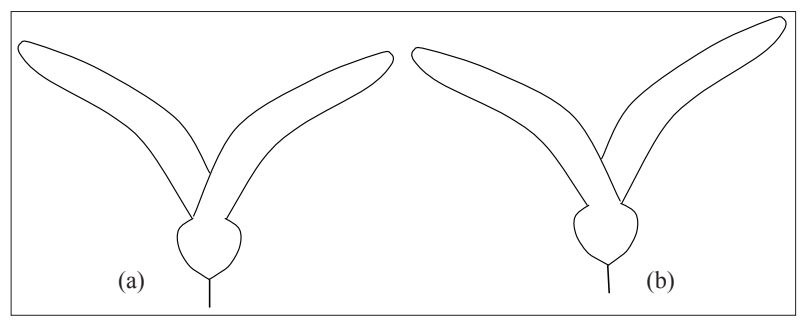

Figure 7: Right-handed (a) and left handed (b) fruits of Dipterocarpus zeylanicus. Other two winged fruits of dipterocarps have a very similar morphology their population in Sri Lanka. Possibly the same applies to other members of this genera, irrespective of the geographical location.

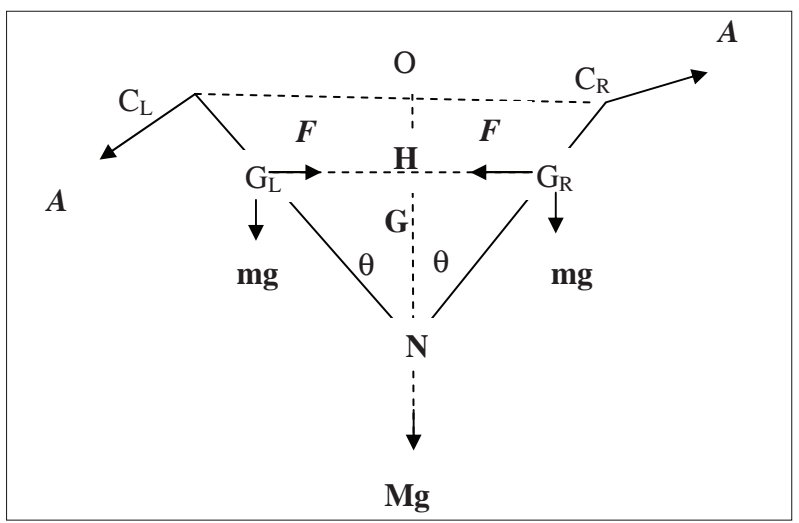

Figure 8: Forces acting on Dipterocarpus fruit and the points of their action: A - aerodynamic forces acting at centres $C_{L}$ and $C_{R}$; F - centrifugal forces acting at $\mathrm{G}_{\mathrm{L}}$ and $\mathrm{G}_{\mathrm{R}} ; \mathrm{mg}$ - weight of the wings acting at $\mathrm{G}_{\mathrm{L}}$ and $\mathrm{G}_{\mathrm{R}} ; \mathrm{Mg}$ - weight of the nut acting at $\mathrm{N}$

The propellers of an airplane or a ship generate a forward thrust when rotated in one sense and backward thrust in the opposite sense of rotation. The trailing edge of the dipterocarp wing is the slightly bulged lobe. Therefore the RH fruit (Figure 7a) will rotate counter-clockwise when viewed from above as it falls, and the LH fruit (Figure 7b) will rotate clockwise.

Forces acting on a falling fruit are the aerodynamic forces $A\left(A_{\mathrm{Z}}, A_{\mathrm{r}}, A_{\varphi}\right)$ acting on the wings at the centres $\mathrm{C}_{\mathrm{L}}$ and $\mathrm{C}_{\mathrm{R}}$; centrifugal forces $F$ acting at the centres of gravities $\mathrm{G}_{\mathrm{L}}$ and $\mathrm{G}_{\mathrm{R}}$ of the wings, weight $m g$ of the wings acting at their centres of gravity $G_{L}$ and $G_{R}$ and weight $M g$ of the nut acting at $\mathrm{N}$ (Figure 8). When the fruit is spinning, angle between the wings $(2 \theta)$ open up a little bit, until it is equilibrated by the elastic forces in the wing and centripetal force. If the fruit is perturbed by tilting inclination from $\theta$ to $(\theta+\varepsilon)$, the resulting motion is about $\mathrm{G}$ described by

$I_{G} \frac{d^{2} \varepsilon}{d t^{2}}=-A_{z} d \varepsilon$

where $d=\mathrm{OG}, s=\mathrm{HG}<\mathrm{d}$ and $l=\mathrm{GN}$. When the terminal velocity has reached $A_{Z}=(\mathrm{M}+2 \mathrm{~m}) \mathrm{g}$ and the equilibrium is stable, provided $d>0$. Thus there exist limitations to mass and area distribution of the wings. Similarly, if the centre of gravity is shifted to either right or left of the line ON, stability would be affected. 
The dynamics governing rotational and translational motion of the stabilised system parallel that of the maple seed and equations (14) - (17) illustrate this. During the decelerating phase of motion, stability gets complicated due to the flexibility of the wing. On loading the nut artificially, a point is reached when the wings do not open and the rotational effect ceases to function- possibly because of the insufficiency of the lift forces or their diversion. In nature this does not happen, unless evolution has turned retrograde due to changing environment. Weight of the wings in relation to the nut and wing dimensions being well balanced, they rarely collapse when falling. Auto-gyrating seeds have evolved to optimise the lift keeping drag to a minimum and also to acquire the terminal value as soon as possible. Just as in maple, the strategy has been to dissipate potential energy via rotation. According to equation (17), larger lift and lower drag coefficients favour smaller terminal velocities. Dipterocarps with heavier nuts have longer, but not so broadened wings. To a good approximation, the terminal motion of a falling dipterocarpus nut can be described by equation (17). It has been suggested that the terminal velocity acquired by wind dispersing seeds vary with the square root of the mass to area ratio (Augspurger \& Frason, 1987; Greene \& Quesada, 2005). Equation (12) incorporates this trend, but more specifically, the parameter ' $l$ ' in equation (13) much more closely the length of the wing. Our experiment with Dipterocarpus zeylanicus showed a variation very close to the square root of the mass to wing length.

\section{Evolution of dipterocarp wings}

Dipterocarps have species with fruits having two, three or five sepals more elongated to form wings. Two winged ones seem to be more abundant compared to three and five winged species and five winged varieties are least abundant. The author could not find any evidence in the literature for existence of species with four wings. Wings have evolved from five sepals centred on the vertices of a pentagon. The following considerations could be a clue as to how the wings could have evolved. If equal weights are placed at the vertices of a regular pentagon with side of length $\mathrm{x}$, the centre of gravity will be: (1) obviously at the centre of the inscribing circle if all the vertices are loaded; (2) $1.4 \mathrm{x}$ from the centre if the vertices 1 , $2,3,4$ or any of the equivalent vertices are loaded; (3) $1.3 \times$ from the centre if the vertices $1,2,3$ are loaded; (4) $\sim 0.88 \times$ from the centre if the vertices $1,3,4$ are loaded; (5) $\sim 1.5 \mathrm{x}$ from the centre if the vertices 1,2 are loaded; (6) $\sim 1.29 \mathrm{x}$ if the vertices 1,3 are loaded. Two winged ones seem to be more abundant compared to three and five winged and five being the least abundant. As a result of inherent handedness in the sepal arrangement in the calyx, fruit variations with longer sepals would spin faster slowing down the descent assisting wind dispersal, and this advantageous trait would have amplified leading to species with five elongated sepals. Although five wings ensure stability, there are number of disadvantages. It increases the weight and also the moment of inertia about the rotational axis, making it harder to rotate in gaining the lift, and both factors diminish the terminal velocity and increase the time elapsed to reach the terminal value. Furthermore, longer wings yield more lift, because the counting factor (velocity) ${ }^{2}$ varies as (wing length) ${ }^{2}$. Increasing the length of the five wings would be too much of a burden to the organism and broader five wings will affect air flow. Thus, the only option available would be to cut-down the number of wings. If a variation produces a variety with four wings, that strain will be inferior because of the lesser degree of stability in falling of the fruit, as the centre of gravity shifts furthest from the centre when the number of wings is four. The next option will be to have three wings in positions 1,2 , and 4 of the pentagon, when the centre of gravity shifts only $0.88 \mathrm{x}$ from the centre. As expected, in three winged species the wings are positioned at vertices 1, 2, 4 of the pentagon. Amazingly, almost all extant and extinct Miocene fossil dipterocarps (Shi \& Li, 2010; Feng et al., 2013) with three wings, have one conspicuously shorter, i.e., if the wings are affixed at the vertices $1,2,4$, the shorter wing is either 1 or 2 . Clearly, this variation adjusts the centre of gravity closer to the centre of the pentagon. Possibly dipterocarps proliferated and became invasive after the origin of three winged species and their successful dispersion by wind. Now the dipterocarps have acquired a genetic facility to control the length of one wing, i.e., a wing in position 1 or 2 in the 1, 2, 4 arrangement. Certainly, species with a very short and almost shunted third wing ( 1 or 2 positions) have emerged, and a trait to

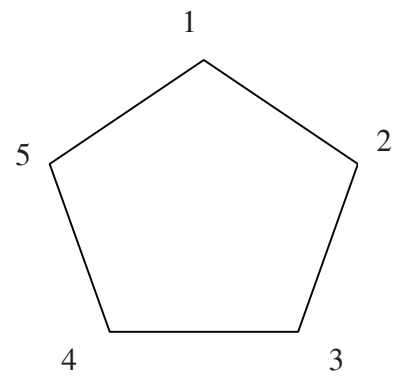

Figure 9: Sepals of dipterocarps are placed at the vertices of a pentagon. The vertices are marked $1-5$ for the purpose of identification of the relative positions of elongated sepals that form wings 
bend wings so as to adjust the centre of gravity evolved subsequently. Developing two wings relieve the tree of the burden of synthesising more biomass and two wings perform better in slowing the falling speed. Generally evolution proceeds by gradual trial and error corrective steps and not by quantum jumps. It would be highly improbable for the two winged species to have evolved in one step by cutting down three wings from the five. The hypothesis we have proposed can be tested by examining the phylogeny of species of Dipterocarpaceae with different number of wings.

The conclusions of some experiments conducted to measure distance of deflection $(D)$ of dipterocarpus seeds suggest that they do not move beyond a few tens of meters from the parent tree, and under conditions of nominal wind speeds and occasional high speed winds may carry them a longer distance (Smith et al., 2015; Song, 2015). The crucial deciding parameter should be the terminal velocity $v_{\mathrm{T}}$ and mere measurement of $D$ does not give the information necessary for determining the dispersal potential. Furthermore, winds have both horizontal and vertically upward components $u_{\mathrm{H}}$ and $u_{\mathrm{v}}$, respectively. As aerodynamic forces are proportional to the square of air speeds relative to a moving object, the terminal velocity in the presence of an updraft of speed $u$ is given by,

$v_{T}=v_{T 0}-u_{V}=\left[\frac{m g}{a^{2}} \frac{C_{\varphi D}}{C_{\phi L} C_{z L}}\right]^{1 / 2}-u_{V}$

where $v_{T 0}$ is the terminal velocity in the absence of the updraft component of the wind. The updraft generates an additional lift and the possibility of $v_{\mathrm{T}}$ becoming negative implies that the nut could even propel a significant distance upwards before $v_{T}$ approaching zero and start descending. Suppose a wind has constant horizontal speed $u_{\mathrm{H}}$ and vertical component $u_{V D \tau}$ greater than $v_{T 0}$ lasting for time $D \tau$ then remaining constant at $u_{V}<v_{\mathrm{T} 0}$. If the canopy height $=h$, the deflection distance will be,

$$
D=u_{H} \Delta \tau+\left[\frac{h+\left(u_{V \Delta \tau}-v_{T 0}\right) \Delta \tau}{\left(v_{T 0}-u_{V}\right)}\right] u_{H}
$$

We found that the average velocity for Dipterocarpus zeylanicus fruits is $v_{T 0} \sim 3 \mathrm{~ms}^{-1}$. Giving the following plausible values for other parameters, i.e., $h=40 \mathrm{~km}, u_{H}=2 \mathrm{~ms}^{-1}, u_{V D \tau}=5 \mathrm{~ms}^{-1}, u_{\mathrm{v}}=1 \mathrm{~ms}^{-1}$, we obtain $D=2.4 \mathrm{~km}$. Dipterocarps have intricately designed wings at much cost to the individual, as such their purpose could not be just for a stone's throw deposition from the parent.
A schematic representation (Figure 10) shows how the wings of a typical dipterocarp are attached to the nut, the orientation and structure of the wing surface. Longitudinal veins covering the surface are more or less straight on one side of the wing and slightly curved diverging on the opposite side of the same wing, and the pattern is reversed in the two wings. During falling the nut rotates around the vertical axis (extension of the straight line segment at the bottom of Figure 10) clockwise as edges of the wings marked with arrows are leading edges. Veins diverge and curve towards the trailing edge as if the veins imprint the flow lines. The wings are also covered with a fine mosaic of veins more concentrated towards the upper end of the wing and the leading edge. We believe that these mosaics serve as vertex confiners to enhance the lift. Wind tunnel experiments have shown that attached leading edge vortices form when a maple seed spins, and the resulting low pressure creates the lift and torque and also provides centrifugal force. It appears that the same mechanism operates in the case of dipterocarps. The angular momentum of the spinning seed is compensated by the vorticity.

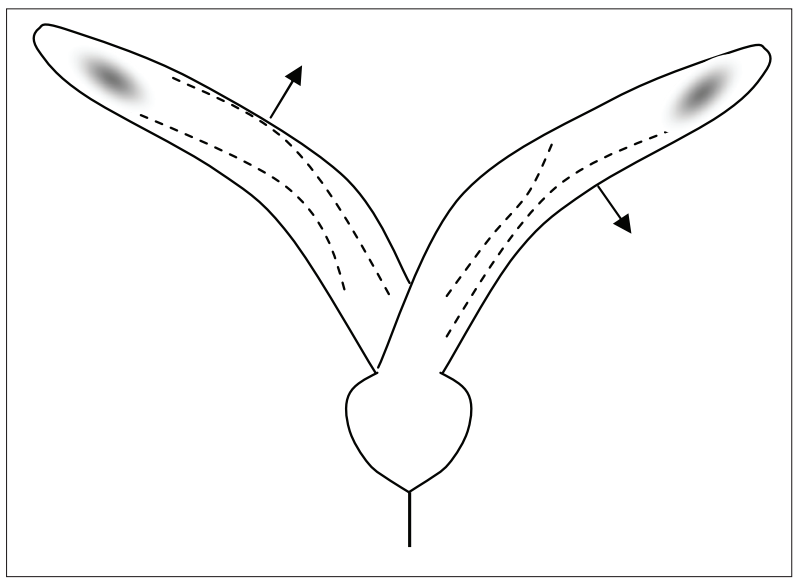

Figure 10: Schematic diagram illustrating the orientation of wings of a typical dipterocarp relative to the nut. Only two veins (dotted lines) drawn to indicate how they diverge, and leading edges are marked with arrows. Wings also have fine mosaic of veins throughout but concentrated near the shaded spots, suggesting that vortices are confined to that region.

\section{Entertaining and intriguing motion of paper helicopters simulating dipterocarps}

To simulate dipterocarp wind dispersal we also examined paper helicopters, which children make and sometimes demonstrated in a wind tunnel in science museums. 
Despite the simplicity of construction, they display the necessity of a handedness. They can be crafted giving a handedness to fix the sense of rotation or to be symmetric and break the R-L symmetry spontaneously.

The toys (a) and (b) shown in Figure 11 are fabricated by cutting a single sheet of paper and folding; the dark rectangle denotes the paper clip used as a weight. They are mirror images of each other and distinct, because there exists no automorphism between them. When dropped and viewed from above (a) rotates clockwise and (b) counter-clockwise. Copter (c) is made by pasting two half glued rectangular strips and folding. Model (c) is $\mathrm{R}-\mathrm{L}$ symmetric, nevertheless will also rotate in one sense other. Careful examination reveals a clear difference of the behaviour of (c) compared to asymmetric models (a) and (b). If care is taken to keep the wings horizontal without twisting, the performance of model (c) is found to be poor and unstable unless weight of the paper clips is increased. Once sufficiently loaded it will fall a longer distance and rotate either clockwise or counter- clockwise breaking the R-L symmetry spontaneously. If any asymmetry is introduced to the wings of the model the performance greatly improves. In models (d) and (e) darkened rectangular portions in the wings have been cut-off, and the performance remarkably improves with (d) rotating counter-clockwise and (e) clockwise. A similar asymmetry can be introduced by twisting the flaps. If a wilful asymmetry is not introduced to the model (c), at heavier loadings the wings twist on its own breaking the symmetry. Our findings agree with the results of similar experiments carried out with plane single and double wings made of paper (Stevenson et al., 2015). Results show that two-wings systems rotate stably and drive a significant lift, provided there is an inherent asymmetry.

\section{Right-left symmetry and wind dispersing conifers}

It was interesting to observe that conifers also possess right-left symmetries though not so conspicuous, but akin to the pattern seen in dipterocarps. Examination

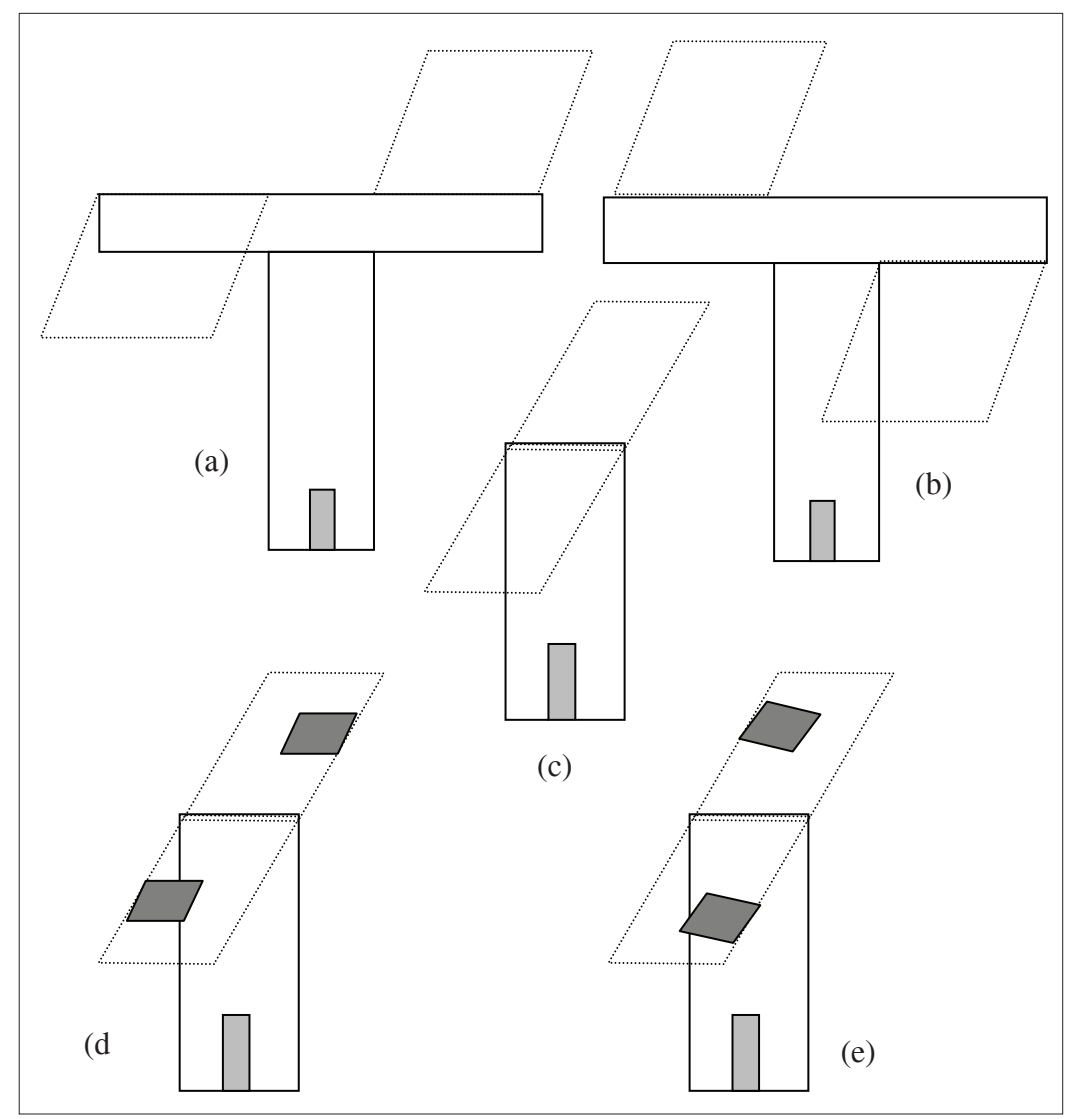

Figure 11: Toy paper helicopters (a) and (b) are mirror images of each other and (c) is symmetric and has no handedness. (d) and (e) are asymmetric models made by removing the shaded rectangular portion from each wing 
of pines revealed that twigs have handedness. The leaf fascicles in pine are arranged as two spirals winding in opposite directions. However, the number of winding turns the spiral takes to reach the same angular position of the fascicle immediately above or below are different in the two spirals, giving a net handedness to the twig. Just like in dipterocarps, the twigs of both kinds are equally distributed. Cones have the same feature, there again, the samaras in the fruit are arranged following the same pattern as the fascicles, so that there are two types of cones, RH or LH born in equal abundance. Samaras are bilaterally asymmetric, but all of them, similar in one kind of cone ( $\mathrm{R}$ or $\mathrm{L}$ ) but the mirror image in the other type ( $\mathrm{L}$ or $\mathrm{R})$. The ventral view of the samaras of $\mathrm{R}$ and L cones of Eastern White Pine (Pinnus strobes) are shown in Figure 12. As both lobes are folded upwards, the objects are geometrically different and cannot be superposed on each other. Wind dispersing species of pine and other conifers generally have more asymmetric seeds.
Right-left symmetry in magnoliaceae and autogyrating samaras of the tulip tree

The R-L symmetry in magnoliaceae resembles that of pines and dipterocarps. Having examined Walsapu

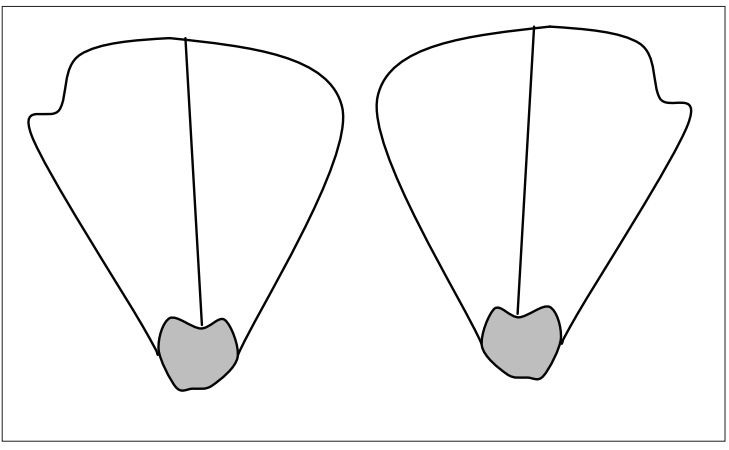

Figure 12: The ventral view of the samaras of right- and lefthanded cones of Eastern White Pine.

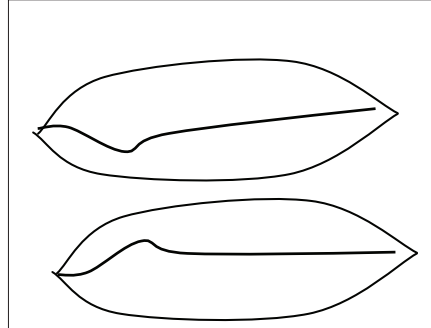

(a)

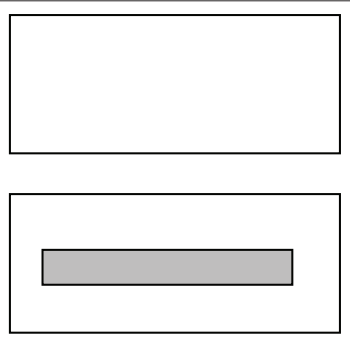

(b)

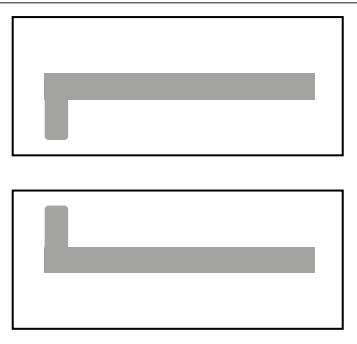

Figure 13: (a) The dorsal view of the samaras of right- and left-handed pods of the tulip tree; (b) rectangular sheets of paper pasted with a letter L cut from paper

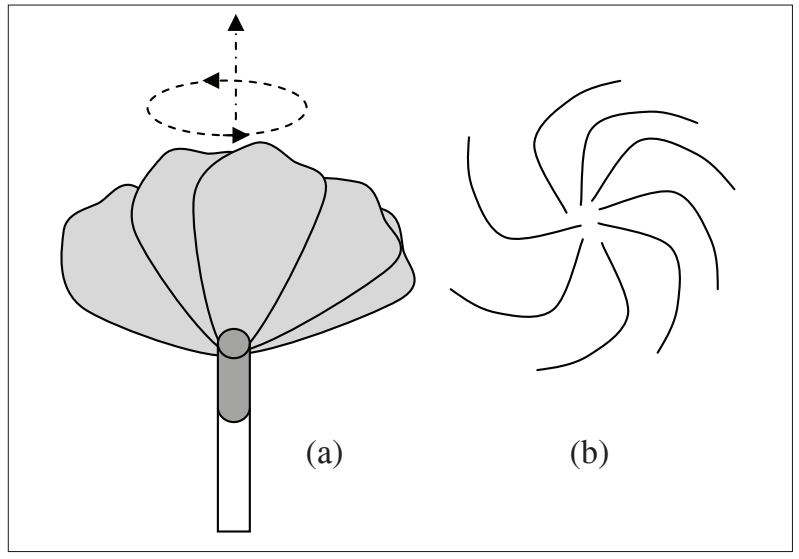

Figure 14: (a) Spinning of a falling Pumeria flower loaded with a match stick; (b) a parachuting seed of Wrighta antidyscenterica as viewed from above
(Michelia nilagrica) and a few related species in Sri Lanka, the tulip tree (Liriodendron tulifera L.) and ornamental magnolias in the United States, we observed that all these genera have twigs of spiral phyllotaxy and R-L helicities occur in equal proportion. Sepals and petals are almost indistinguishable but the handedness of the twig passes on to the flower and the fruit. In flower buds two opposite spirals are seen (normally 5/8 Fibonnacci) correlated to the sense of leaf phyllotaxy. Just as in dipterocarp and pine there are $\mathrm{R}$ and $\mathrm{L}$ fruits. The $\mathrm{R}(\mathrm{L})$ fruit of the tulip tree has $\mathrm{R}(\mathrm{L})$ samaras readily distinguishable from the direction of twist in the ridge placed midway and in the lengthwise direction (Figure 13a). Samaras of $\mathrm{R}$ and $\mathrm{L}$ pods are mirror images of each other and no automorphism exists because of ventral-dorsal difference. During descent (a) and (b) gyrate in opposite senses with middle ridge 
as the axis. R- L asymmetry provides torque for the rotational motion, creating a lift. The moment of inertia is minimum about the mid axis and motion remains stable. It has been suggested that this stability greatly helps dispersal, although the terminal velocities attained are smaller compared to maple (Mccutchen, 1977).

We have also conducted simple paper toy experiments to simulate the auto-gyration of tulip tree samaras. Figure 13(b) depicts four rectangles $\left(\sim 2.5 \times 10 \mathrm{~cm}^{2}\right)$ cut out of ordinary thin writing paper. In one of them, a thin rectangular strip of paper is pasted symmetrically and the remaining two with pasted letter L cut from the same paper as shown. Because of the thickness of the letter L pasted, the two configurations in 13(b) with the letter $\mathrm{L}$ are distinct objects and mirror images of each other, and the first two have handedness. When dropped from a height all four glide and rotate with length-wise side remaining nearly horizontal but with the following differences: the symmetric ones rotate in either sense when viewed from a fixed direction relative to the rectangle; however, the one with the middle ridge is much more stable; the ones pasted with letters L rotate in opposite directions. A simple experiment sometimes gets complicated owing to the twist developed when the strips are pasted. Many experiments have been performed to study the aerodynamics of falling paper, cards and discs and the general conclusion has been that they are tumbling and unstable (Pesavento \& Wang, 2004; Changqiu \& Xu 2008; Varshney et al., 2013). We have found that the mid ridge greatly stabilises the motion. Another important difference we observed in our experiments with falling thinner flexible paper rectangles is the stability and absence of tumbling. The scaling of these systems depend not only on the aspect ratios and the Reynolds number, when elastic deformation enter into the picture. If the length of the bare rectangle is increased to $16-18 \mathrm{~cm}$, the paper rotates very stably in horizontal orientation and both senses of rotation is observed with near equal probability. Here, the paper rectangle during motion spontaneously acquires handedness because of the elastic deformation and spin stably. The effect could have important implications in biological systems.

\section{The case of apocyanaceae}

One of most unusual chirality features are seen in apocyanaceae. In almost all genera we examined that signs of a handedness is not seen in phyllotaxy or branching. However, flowers of the entire family (we have not seen exceptions) are imbricated in the same sense (left-handed, according to the convention we have adopted). An entertaining way to see this would be to load an apocyanaceae flower (e.g. Pumeria L.) stalk with a match stick and let it fall. Always they rotate counterclockwise when viewed from above (Figure 14a).

A majority of apocyanaceae has no edible fruits, instead they contain poisonous alkaloids. Flowers are fragrantly scented and heavily loaded with nectar, suggesting that their ancestry has been in the insect era well before the birds. Many species of apocyanaceae have plumed seeds dispersed by wind. The left-handedness of the family transcends to fruits and seeds. As a result of the chirality parachuting seeds slowly rotate. Here the advantage of rotation seems to be not the generation of a lift, but an enhancement of the drag. The centripetal force of rotation spreads the plume in the horizontal plane increasing the drag. The appearance of the long hairs in a parachuting Wrighta (Wrighta antidyscenterica) is shown in Figure 14(b).

\section{On origin of handedness in plants}

Recent experiments suggest that auxins trigger spiral positioning of leaves (Reinhhardt et al., 2003; Niklas et al., 2006; Kramer, 2008; Chitwood, 2012). However, what determines the sign of handedness remains unanswered. The leaves of tomato have been made to break the bilateral symmetry by application of auxins to the leaf primordium. A rare example of a herb conspicuously displaying breaking of bilateral symmetry is Agalonema pseudo-bracteatum (Tennakone \& Iqbal, 1998), where right and left-handed lobes greatly differ in area and disparity is higher at seasons of faster growth. These are clearly amplification effects of an already existing spiral phyllotaxy.

Leonardo da Vinci noted that the cross-sectional area of a tree trunk approximates to the sum of areas of branches as if growth is like the flow of an incompressible fluid (Richter, 1970). Based on the idea, we give an explanation for auxins effect as follows:

The Navier Stoke equation in vorticity form reads,

$$
\frac{\partial \omega}{d t}=\nabla \times(u \times \omega)+\frac{1}{R_{e}} \nabla^{2} \omega
$$

where $u$ = fluid velocity, $\omega=\Delta \times u$ and $R_{e}=$ Reynolds number

The vorticity equation (22) can also be cast into the form $(u \times \omega \neq 0)$ 


$$
\frac{\partial \omega}{d t}=\omega . \nabla u-u . \nabla \omega+\frac{1}{R_{e}} \nabla^{2} \omega
$$

We make the reasonable assumption that $\nabla u=G$ and $\omega$ are in parallel directions (growth direction), and $u$ and $\nabla \omega$ are orthogonal. Thus equation (23) approximates to a reaction diffusion equation,

$$
\frac{\partial \omega}{d t}=\omega G+\frac{1}{R_{e}} \nabla^{2} \omega
$$

and auxins that create large growth gradients enhance the spirality generated spontaneously or as a result of bias.

\section{Practical significance - biomimetic aerodynamic innovations}

Aerodynamic designs are based on powerful but yet imperfect theory and experimentation. Although evolutionary corrections have been incorporated, optimisation via testing varying models is prohibitively time consuming and expensive. Wind dispersing seeds are the aerodynamic systems most extensively optimised via trial and error based selection. Consequently, they are expected to possess favourable attributes blind to modern engineering. An important implication of the above work, is that auto-gyrating seeds have successfully achieved a design perfection to divert drag forces to generate rotation, increasing the lift. Wing shape and striations of veins seem to play an important role. Veins play a dual role as the structure supporting the wing and optimised air flow. These may be incorporated to build light-weight but structurally strong airfoils and rotor blades. Remotely controlled, light weight small airplanes and helicopters consuming minimal quantities of fuel find peaceful applications in agriculture and aerial photography. Similarly, solar powered air planes need new designs to bear the weight of the cells and compromise light harvesting and lift.

Even a simple observation related to wind dispersal of seeds could have profound implications on practical aerodynamics. As pointed out earlier Box Elder samaras have a distinctly different wing shape compared to the closely related other maple species, which are taller. Experiments indicated that Box Elder samaras acquire the terminal velocity in a comparatively shorter duration. Theoretical understanding of the distinctions in wing structures of maple species might lead to new airfoil and rotor designs. Well preserved fossil winged dipterocarp seeds have been discovered (Shi \& Li, 2010), and careful study of their structure in comparison to extant species could yield clues to variations resulting from climate change.

\section{CONCLUSION}

Maple and dipterocarps are two tree species, most successfully proliferated owing to the evolution of seeds with wing appendages. Wings effectively slow down the gravitational descent via generation of a rotational lift. A theoretical formalism was developed to explain the observed motion, account for the stability and calculate terminal velocities - the crucial parameter determining the deflection of seeds during wind dispersal. Results indicate that under favourable conditions, these seeds can disperse kilometre distances.

Despite the similarity of the basic aerodynamics, a distinct difference exists between maple and dipterocarps. Maple samaras possess no handedness; consequently they spin either clockwise or counter-clockwise in descending, as a result of spontaneous breaking of the symmetry. However, systems exhibiting this behaviour are highly susceptible even to the smallest biasing perturbation. The factors inducing such biases were identified and experiments indicate that although individual seeds frequently violate the symmetry, globally it is respected. It is not possible to quantify the variations in maple seed attributes and correlate them to a bias in the sense of rotation, however frequency analysis reveals normal distribution indicative of no preference. In contrast dipterocarpus nuts possess a natural handedness and a geometrical difference can be identified to a confidence level more than $99 \%$. Here, both right and left handed fruits are produced in equal abundance, and during falling rotate in opposite directions. The investigation disclosed that the maple species have no organs with right-left distinguishing attributes whereas in dipterocarps, the twigs, racemes, flowers and sepals have handedness. The sepals are placed in a twisted arrangement at the vertices of a pentagon. Sepals elongate to form either five, three or two wings excluding four, and majority of the species have only two wings. This is explained as an evolutionary reaction to the centre of gravity instabilities, aerodynamic effectiveness and the natural requirement of reducing burden biomass production. Likewise the R-L asymmetry of pine cones and samaras associate itself with the handedness of the leaf spiral phyllotaxy. Conifers and some genera of magnoliaceae also possess R-L asymmetry characters similar to dipterocarps. In apocyanaceae, unlike dipterocarps and conifers, the phyllotaxy does not display handedness but the flowers are always left-handed and this asymmetry transcends to the fruit and seeds. Several genera of apocyanaceae have plumed seeds dispersed by wind. Here the advantage of handedness seems to be spreading of hairs of parachuting seeds by centrifugal acceleration, thereby enhancing the air drag. 
Wind dispersing seeds in plants of widely separated families adopt auto-gyration as an effective mechanism of slowing down the descent. Auto-gyration fundamentally necessitates a breaking of the R-L symmetry. Maple has designed the seeds to achieve this as a dynamically generated SBS. In dipterocarps, magnolias and pines, a morphologically induced SBS endows seeds with a handedness, either right or left. Whereas in apocyanaceae, presumably a persisting accidently introduced genetic R-L disparity induce unique chirality of the seeds. Another possibility would be to generate an asymmetry to the seed appendage via a spontaneous asymmetric deformation, as a consequence of motion. It is not impossible that this method is also realised somewhere in wind dispersal. Apart from a requirement of morphogenesis, it is hard to conceive the advantage of a handedness to a plant in any of its functions other than wind dispersing. Plants even in families widely separated, possessing R-L distinctive characteristics have exploited it. Wind dispersal have utilised the available traits and optimised in the course of evolution (Wrights et al., 2008); handedness is one such trait.

\section{Acknowledgement}

A significant portion of this investigation is based on the knowledge gained while conducting the research project 'Right-Left Symmetry in Plants (RG/79/21)' supported by the Natural Resources, Energy and Science Authority of Sri Lanka (NARESA). The author also gratefully acknowledge the assistance granted by the staff, Department of Physics and Department of Biology, University of Sri Jayewardenepura (1977-1980) and the Department of Physics, University of Ruhuna (1982 1988).

\section{REFERENCES}

1. Ashton P.S. (1980). Dipterocarpace. A Revised Handbook to the Flora of Ceylon (eds. M.D. Dassanayake \& F.R. Fosberg), volume 1, pp. 364 - 423. Amerind Publishing Company, New Delhi, India.

2. Ashton P.S. \& Gunatilleke C.V.S. (1987). New light on plant geography of Ceylon. Journal of Biogeography 14: $248-285$.

DOI: https://doi.org/10.2307/2844895

3. Augspurger C.K. \& Frason S.E. (1987). Wind dispersal of artificial fruits, varying in mass, area and morphology. Ecology 68: 27 - 42.

DOI: https://doi.org/10.2307/1938802

4. Bagheri S., Mazzino A. \& Bottarol A. (2012). The spontaneous symmetry breaking of a hinged flapping filament generates lift. Physical Review Letters 109: $154502-15405$.
DOI: https://doi.org/10.1103/PhysRevLett.109.154502

5. Carlton J. (2007). Marine Propellers and Propulsion. Butterworth-Heinemann Elsevier Ltd., London, UK.

6. Changqiu J. \& Xu K. (2008). Numerical study of the unsteady aerodynamics of freely falling plates. Communication in Computational Physics 3: 834 - 851.

7. Chitwood D.H., Headland L.R., Ranjan A., Martinez Braybrook S.A., Koenig D.P., Kuhlemeier C., Smith R.S. \& Sinha N.R. (2012). Leaf asymmetry as a developmental constraint imposed by auxin-dependent phyllotactic patterning. The Plant Cell 24: $1-10$.

DOI: https://doi.org/10.1105/tpc.112.098798

8. Corlett R. \& Primack R. (2005). Dipterocarps: trees that dominate the Asian rain forest. Arnoldia 63(3): 3 - 7. Available at http://people.bu.edu/primack/Dipterocarps. $p d f$, Accessed 22 November 2015.

9. Dayanandan S., Ashton P.S., Williams S.M. \& Primack R.B. (1999). The phylogeny of the tropical tree family dipterocarpaceae based nucleotide sequence of the chloroplast RBCLgene. American Journal of Botany 86: $1182-1190$.

DOI: https://doi.org/10.2307/2656982

10. Feng X., Tang B., Kodrul T.M. \& Jin J. (2013). Winged fruits and associated leaves of Shorea (Dipterocarpaceae) from Late Eocene of South China and their phytographic and paleoclimatic implication. American Journal of Botany 100: $574-581$.

DOI: https://doi.org/10.3732/ajb.1200397

11. Foxworthy F.W. (1911). Philippine Dipterocarpaceae. Manila Bureau of Printing, Manila, The Philippines.

12. Greene D.F. \& Quesada M. (2005). Seed size, dispersal, and aerodynamic constraints within the Bombacacea. American Journal of Botany 92: 998 - 1005.

DOI: https://doi.org/10.3732/ajb.92.6.998

13. Gunatilleke C.V.S., Gunatilleke I.A.U.N., Esufali S., Harms K.E., Ashton P.M.S., Burslem D.F.R.P \& Ashton P.S. (2006). Species-habitat associations in a Sri Lankan dipterocarp forest. Journal of Tropical Ecology 22: 371 384.

DOI: https://doi.org/10.1017/S0266467406003282

14. Hederstrom A. (2002). Aerodynamics and evolution of avian flight. Trends in Ecology and Evolution 13: 415 422.

15. Kramer E. (2008). Computer models of auxin transport: a review and commentary. Journal of Experimental Botany 59: $45-53$.

DOI: https://doi.org/10.1093/jxb/erm060

16. Lamarque L.J. (2013). Ecology and evolution of invasive maple tree species. PhD thesis, York University, Toronto, Canada.

17. Lentink D., Dickson W., van Leeuwen J. \& Dickinson M.H. (2009). Leading-edge vortices elevate lift of autorotating plant seeds. Science 324: 1438 - 1440.

DOI: https://doi.org/10.1126/science.1174196

18. Lugs H.J. (1983). Annual Review of Fluid Mechanics 15 : $123-147$.

DOI: https://doi.org/10.1146/annurev.fl.15.010183.001011

19. McCutchen C.W. (1977). The spinning rotation of ash and 
tulip tree samara. Science 197: $691-692$.

DOI: https://doi.org/10.1126/science.197.4304.691

20. Narasimhan N. (2015). Avenue de Henri Benard, http:// www.nanoscience.info/, Accessed 01 December 2015.

21. Niklas K.J., Spatz H.C. \& Vincent J. (2006). Plant biomechanics: an overview and prospectus. American Journal of Botany 93: 1369 - 1378. DOI: https://doi.org/10.3732/ajb.93.10.1369

22. Pandolfi C. \& Izzo D. (2013). Biomimetics on seed dispersal: survey and insights for space exploration. Bioinsspiration and Biomimetics 8: 025003 - 025012. DOI: https://doi.org/10.1088/1748-3182/8/2/025003

23. Pesavento U. \& Wang J.Z. (2004). Falling paper: NavierStoke solutions, model of fluid forces and centre of mass elevation. Physical Review Letters 93: 144501 - 14404. DOI: https://doi.org/10.1103/PhysRevLett.93.144501

24. Pope A. (2010). Basic Wing and Air Foil Theory. Dover Publications, Mineola, New York, USA.

25. Reinhhardt D., Pesce E.R., Stieger P., Mandel T., Baltensperger K., Bennet M., Trass J., Friml J. \& Kuhlemeier C. (2003). Regulation of phyllotaxis by polar auxin transport. Nature 426: 255 - 260.

DOI: https://doi.org/10.1038/nature02081

26. Richter J.P. (1970). The Notebooks of Leonardo da Vinci $(1452$ - 1519), compiled and edited from the original manuscripts. Dover Publications, Mineola, New York, USA.

27. Sane S.P. (2003). Aerodynamics of insect flight. Journal of Experimental Biology 206: 4191 - 4208. DOI: https://doi.org/10.1242/jeb.00663

28. Shi G. \& Li H. (2010). A fossil fruit wing of Dipterocarpus from middle of Fujian, China and its palaeoclimatic significance. Review Palaeobotany and Palynology 162: $559-606$.

DOI: https://doi.org/10.1016/j.revpalbo.2010.08.001

29. Smith J.R., Bagchi R., Ellens J., Kettle C.J., Burslem D.F.R.P., Maycock C.R., Khoo E. \& Ghazoul F. (2015). Predicting dispersal of auto-gyrating fruit in tropical trees: a case study from Dipterocarpaceae. Ecology and Evolution 5(9): $1794-1801$.

DOI: https://doi.org/10.1002/ece3.1469

30. Smitinand T., Sanitiusk T. \& Phengklai C. (1980). The manual of Diptercapaceae of Mainland South East Asia. Thai Forest Bulletin of Botany 12: 1 - 37.
31. Simmathri A. \& Turnbull J.M. (1998). A Review of Dipterocarps, Taxonomy, Ecology and Silviculture. Forest Research Institute, Kepong, Malaysia.

32. Sohn M.H., Lee E.J. \& Sohn M.H. (2014). Mechanism of autorotation flight of maple samaras (Acer palmatum). Experiments in Fluids 55: 1718 - 1726. DOI: https://doi.org/10.1007/s00348-014-1718-4

33. Song K.W.(2015). The kinematics of a falling Dipterocarpus Alatus seed. International Scholastic Journal of Science 9: $1-6$.

34. Stevenson R.A., Evangelista D. \& Looy C.V. (2015). When conifers took: a biomechanical evaluation of an imperfect evolutionary takeoff. Paleobiology 41: 205 - 225. DOI: https://doi.org/10.1017/pab.2014.18

35. Tennakone K., Dayatilleke R.K.D. \& Ariyaratne I. (1982). Right-left symmetry and phyllotaxy and imbrication of the flowers of Hibiscus furcartus L.. Annals of Botany 50: 397 -400 .

DOI: https://doi.org/10.1093/oxfordjournals.aob.a086379

36. Tennakone K. \& Iqbal M.C.M. (1998). Breaking of right-left symmetry in leaves of Agalonema pseudobractetum, Proceedings of the Sri Lanka Association for the Advancement of Science, part I, 14 - 19 December, Sri Lanka Association for the Advancement of Science, Colombo, p. 260.

37. Varshney K., Chang S. \& Wang Z.J. (2012). The kinematics of falling maple seeds and initial transition to helical motion. Nonlinearity 25: $\mathrm{C} 1-\mathrm{C} 8$. DOI: https://doi.org/10.1088/0951-7715/25/1/C1

38. Varshney K., Chang S. \& Wang Z.J. (2013). Unsteady aerodynamic forces and torques on falling parallelograms in coupled tumbling-helical motion. Physical Review E 87: $053021-053026$.

DOI: https://doi.org/10.1103/PhysRevE.87.053021

39. Wrights S.J., Trahtenbrotb A., Bohrerc G., Dettod M., Katule G.G., Horvitzb N., Muller-Landaua H.C., Jonesa F.A. \& Nathanb R. (2008). Understanding strategies for seed dispersal by wind under contrasting atmospheric conditions. Proceedings of the National Academy of Science 105: 19084 - 19089.

DOI: https://doi.org/10.1073/pnas.0802697105

40. Zhang J. \& Childress S. (2005). Symmetry breaking leads forward flapping flight. Journal of Fluid Mechanics 506: $147-155$. 